\title{
The effect of prey refuge on the dynamics of three species food web system
}

\author{
Dawit Melese ${ }^{1, *}$ and Abraha Hailu ${ }^{2}$ \\ ${ }^{1}$ Bahir Dar University, Department of Mathematics, Bahir Dar, Ethiopia \\ ${ }^{2}$ Arba Minch University, Department of Mathematics, Arba Minch, Ethiopia
}

\begin{abstract}
In this paper, a mathematical model is proposed to study the effect of prey refuge on the dynamics of three species food web system. The food web comprises of a single prey and two competing predators. The two predators predate their prey following Holling type II functional response. In this work we discussed boundedness of the system, existence condition of the equilibrium points and the Jacobean matrix is obtained by linearization techniques. The local stability of the equilibrium points was discussed by using Routh-Hurwitz criteria and the global stability of the equilibrium points by constructing suitable Lyapunov function. Numerical simulation is conducted to support the analytical result. Finally, the effect of prey refuge on the dynamics of one prey two predator was discussed based on the analytical and numerical simulation results. From the numerical simulations, it is found that the dynamical system is persistent for a small value of the refuge constant. However, an increase in the refuge constant leads to the extinction of one of the predator species.
\end{abstract}

Keywords: Food web; Linearization; Prey refuge; Stability.

DOI: https://dx.doi.org/10.4314/ejst.v14i2.2

\section{INTRODUCTION}

The dynamic relationship between predators and their prey has long been and will continue to be one of the dominant themes in both ecology and mathematical ecology due to its universal existence and importance (Berryman, 1992). Systems of differential equations have a certain extent, successfully to describe the interactions (relationship) between species. The basic system is the Lotka-Volterra model, which models the interaction between a predator and a prey. Various dynamical relations between predators and their prey in ecology and mathematical ecology have been studied (Kuang and Beretta, 1998). The three species interaction shows very complex dynamical behavior (Gakkhar et

\footnotetext{
* Corresponding author: dawitmpg@gmail.com

(C) This is an Open Access article distributed under the terms of the Creative Commons Attribution License (http://creativecommons.org/licenses/CC BY4.0)
} 
al., 2007; Naji and Balasim, 2007; Lv and Zhao, 2008; Upadhyay and Naji, 2009; Yu and Zhao, 2009; Reddy and Ramacharyulu, 2011; Li et al., 2014; Hsu et al., 2015; Panday et al., 2018; Mishra and Raw, 2019). The co-existence and extinction of interacting species have been of great importance and studied extensively in the past (Dubey and Upadhyay, 2004; Naji and Balasim, 2007; Feng et al., 2009; Alebraheem and Abu-Hasan, 2012). Dubey and Upadhyay (2004) have studied the dynamics of one-prey two-predator system with ratio-dependent functional response. Criteria for local stability, instability and global stability of the nonnegative equilibrium points were obtained.

Hsu (1982) proposed and analyzed a model of two predators competing for a single prey. He showed that if the interference coefficient is small, then the winner in purely exploitative system competes its rival successfully and if the interference coefficient is large enough, then the competition outcome depends on the initial population of predator species. Freedman (Freedman and Waltman, 1984) considered three level food webs and two competing predators feeding on a single prey and a single predator feeding on two competing prey species. They obtained criteria for the system to be persistent. Kar (2005) studied on the stability analysis of a prey predator model incorporating a prey refuge.

Jawdat and Yahya (2012) studied two competing predators sharing one prey in homogeneous environment with Holling type-II functional response. The conditions of coexistence and extinction of the predators in the case of non-periodic solution were obtained in terms of efficiency of predator conversion of prey biomass into predator offspring. Edwin (2010) study the dynamics of a system of two prey and one predator in which the predator shows a Holling type II response to one prey that is also harvested, and a ratio-dependent response to the other prey. The major observation from results of numerical simulation is that the predator population density increased significantly when the intrinsic growth rate of both preys increased. This can imply that a high intrinsic growth rate of the prey initially increases their population density, which increases the predator chance of capturing the prey, and so the predator population density increases.

Nature can provide some degree of protection to a given number of prey populations by providing refuges. Such refugia can help in prolonging prey predator interactions by reducing the chance of extinction due to predation (Huang et al., 2006; Kar, 2005; Srinivasu and Gayatri, 2005) and damp prey predator oscillations (Collings, 1995). The effects of prey refuges on the population dynamics are very complex in nature, but for modeling purposes, it can be considered as constituted by two components: the first effects, which 
affect positively the growth of prey and negatively that of predators, comprise the reduction of prey mortality due to decrease in predation success. The second one may be the trade-offs and by-products of the hiding behavior of prey which could be advantageous or detrimental for all the interacting populations (González-Olivares and Ramos-Jiliberto, 2003).

In the literature studies show that refuges have both stabilizing (Hassell, 2020) and destabilizing effect (McNair, 1986). The traditional ways in which the effect of refuge used by the preys has been incorporated in predator prey models is to consider either a constant number or a constant proportion of the prey population being protected from predation (Smith, 1978). Hassel (Hassell, 2020) notes that in reality refugia fall between these two extremes. It is pointed out that those protecting a proportion of the prey population appearing to be more common (Collings, 1995). However, the refuges, which protect a constant number of preys, have a stronger stabilizing effect on population dynamics than the refuges, which protect a constant proportion of prey (González-Olivares and Ramos-Jiliberto, 2003; Krivan, 1998). For more biological background and results on the effects of a prey refuge, one could refer to several other reports (McNair, 1986; Sih, 1987; Collings, 1995; Krivan, 1998; Kar, 2005; Ko and Ryu, 2006).

In this paper, we intended to improve Jawdat Alebraheem and Yahya Abu-Hasan (2012) study on one prey and two-predator system by incorporating prey refuge and taking the classical Holling type II numerical response on the two predators to study the co-existence, boundedness and stability of the system and see the effect of prey refuge on the dynamics of the proposed system.

The organization of the paper is as follows. In section two, the mathematical model is presented. Section three is devoted to mathematical analysis: existence and boundedness of the solutions, existence and stability of the unique steady state of the system. In section four, numerical simulations are shown. At last, conclusion is presented in section five.

\section{Model formulation}

Jawdat Alebraheem and Yahya Abu-Hasan (2012) considered the case of an environment where there is a prey and two predators. A two-predator one prey model was studied with a prey population $X(t)$ and predator populations $Y(t)$ and $Z(t)$. The predators prey on the prey species based on the Holling type-II functional response. Portion of the prey species is supposed to take refuge. The prey species are assumed to grow logistically. Interspecific competition among 
the predator species was also included in the model. The model has had three non-linear autonomous ordinary differential equations describing how the population densities of the three species would vary with time.

The Model equation is written as

$$
\begin{aligned}
& \frac{d X}{d T}=r X\left(1-\frac{X}{K}\right)-F_{1}(X) Y-F_{2}(X) Z \\
& \frac{d Y}{d T}=e_{1} F_{1}(X) Y-\bar{\beta}_{1} Y Z-\delta_{1} Y \\
& \frac{d Z}{d T}=e_{2} F_{2}(X) Z-\bar{\beta}_{2} Y Z-\delta_{2} Z
\end{aligned}
$$

for $X(0)=X_{0} \geq 0, Y(0)=Y_{0} \geq 0, Z(0)=Z_{0} \geq 0, F_{i}(X)=\frac{a_{i} X}{b_{i}+X}, i=1,2$.

It is assumed that prey grows logistically in the absence of predators such that $r$ is the per capita intrinsic growth rate of prey and with carrying capacity $K$. $\bar{\beta}_{1}$ and $\bar{\beta}_{2}$ measure the inter-specific competition of the predator $Z$ on predator $Y$ and vice-versa; $b_{1}$ and $b_{2}$ are the saturation value of the functional response of the predator $Y$ and predator $Z$, respectively. And $a_{1}$ and $a_{2}$ measure efficiency of the searching and the capture of predators $Y$ and $Z$, respectively; $\theta_{1}$ and $\theta_{2}$ represent efficiency of converting consumed prey into predator offspring. $\delta_{1}$ and $\delta_{2}$ are the death rate of predators $Y$ and $Z$, respectively. The functions $F_{I}(X)$ and $F_{2}(X)$ represent the Holling type-II functional responses. The constant $m$ represents prey refuge constant.

In this work, we intended to modify the model (1) by incorporating a refuge protecting $m X$ of the prey. This leaves $(1-m) X$ of the prey available to the predators. Thus, the modified system becomes:

for

$$
\begin{aligned}
& \frac{d X}{d T}=r X\left(1-\frac{X}{K}\right)-F_{1}(X) Y-F_{2}(X) Z \\
& \frac{d Y}{d T}=e_{1} F_{1}(X) Y-\bar{\beta}_{1} Y Z-\delta_{1} Y \\
& \frac{d Z}{d T}=e_{2} F_{2}(X) Z-\bar{\beta}_{2} Y Z-\delta_{2} Z
\end{aligned}
$$

$X(0)=X_{0} \geq 0, Y(0)=Y_{0} \geq 0, Z(0)=Z_{0} \geq 0, F_{i}(X)=\frac{a_{i}(1-m) X}{b_{i}+(1-m) x}, i=1,2$,

where all the parameters in the model are assumed to be positive.

The following non-dimensional state variables and parameters are chosen.

$x=\frac{X}{K}, y=\frac{Y}{K}, Z=\frac{Z}{K}, t=r T_{x} \quad \alpha_{1}=\frac{b_{1}}{K}, \alpha_{2}=\frac{b_{2}}{K}, \beta_{1}=\frac{\beta_{1} K}{r}, \beta_{2}=\frac{\beta_{2} K}{r}, c_{1}=\frac{a_{1}}{r}$, $c_{2}=\frac{a_{2}}{r}, d_{1}=\frac{\delta_{1}}{r}, d_{2}=\frac{\mathbb{E}_{2}}{r}$.

The model equation (2) takes the following non-dimensional form 


$$
\begin{gathered}
\frac{d x}{d t}=x(1-x)-\frac{c_{1}(1-m) x y}{\alpha_{1}+(1-m) x}-\frac{c_{2}(1-m) x z}{\alpha_{2}+(1-m) x}=f(x, y, z) \\
\frac{d y}{d t}=\frac{e_{1} c_{1}(1-m) x y}{a_{1}+(1-m) x}-\beta_{1} y z-d_{1} y=g(x, y, z) \\
\frac{d z}{d t}=\frac{e_{2} c_{2}(1-m) x z}{a_{2}+(1-m) x}-\beta_{2} y z-d_{2} z=h(x, y, z) \\
\text { for } x(0)=x_{0} \geq 0, y(0)=y_{0} \geq 0, z(0)=z_{0} \geq 0 .
\end{gathered}
$$

\section{MATHEMATICAL ANALYSIS}

\section{Positivity and boundedness of the model}

In the theory of the dynamics of food web system, the positivity and boundedness of the system implies that the system is biologically well behaved. One can easily prove that the function $\boldsymbol{f}=(f, g, h)$ of the system (3) is Locally Lipschitz on the region $\Omega=\{(x, y, z) ; x \geq 0, y \geq 0, z \geq 0\}$. Fundamental theorem of existence and uniqueness assures existence and uniqueness of solution of the system (3) with the given initial condition. The uniqueness and existence theorem ensures that any trajectory starting from the first octant remains in it, that is, no trajectory will cross the coordinate axes.

Theorem 1: All the solutions of system (3) which start in $\mathbf{R}_{+}^{3}$ are uniformly bounded.

Proof. Let $x(t), y(t)$ and $z(t)$ be any solutions of the system (3) with positive initial condition.

Let $w=x+\frac{1}{e_{1}} y+\frac{1}{e_{2}} z$.

Then, we have $\frac{d w}{d t}=\frac{d x}{d t}+\frac{1 d y}{e_{1} d t}+\frac{1 d z}{e_{2} d t}$. From equation (3), we have

$\frac{d w}{d t}=x(1-x)-\frac{c_{1}(1-m) x y}{a_{1}+(1-m) x}-\frac{c_{2}(1-m) x z}{a_{2}+(1-m) x}+\frac{c_{1}(1-m) x y}{a_{1}+(1-m) x}-\frac{\beta_{1} y z}{e_{1}}-\frac{d_{1} y}{e_{1}}+\frac{c_{2}(1-m) x z}{a_{2}+(1-m) x}$

$$
\begin{aligned}
& -\frac{\beta_{2} y z}{e_{2}}-\frac{d_{2} z}{e_{2}}, \\
& \leq-x-\frac{d_{1} y}{e_{1}}-\frac{d_{2} z}{e_{2}}-(x-1)^{2}+1, \\
& \leq-\theta w+1,
\end{aligned}
$$

where $\theta=\min \left(1_{1}, d_{1}, d_{2}\right)$.

Thus, we have $\frac{d w}{d t}+\theta w \leq 1$.

Applying the theory of differential inequality, we obtain

$$
0<w(x, y, z) \leq \frac{1-e^{-\theta t}}{\theta}+w(x(0), y(0), z(0)) e^{-\theta t},
$$


which upon letting $t \rightarrow \infty$ yields $w\left(x_{v}, y_{z} z\right) \leq \frac{1}{\theta}$. Therefore, the solutions of the system are bounded for $\eta>0$ and $w(x, y, z)<\frac{1}{\theta}+\eta$.

Hence, all the solutions of (3) that initiate in $\Re_{+}^{3}$ are confined in the region $S=\left\{(x, y, z) \in \Re_{+}^{3} s w(x, y, z)<\frac{1}{\theta}+\eta\right\}$ for any $\eta>0$.

\section{Existence of equilibrium points of the system}

In this section, conditions for the existence of the equilibrium points of the system (3) are established. One can see that the system (3) has five biologically feasible equilibrium points:

1. The trivial equilibrium point: $E_{0}(0,0,0)$,

2. The axial equilibrium points: $E_{1}(1,0,0), E_{2}\left(x^{*}, 0, z^{*}\right), E_{3}\left(\bar{x}_{v}, \bar{y}, 0\right)$,

where $x^{*}=\frac{d_{2} \omega_{2}}{\left(\varepsilon_{2} c_{2}-d_{2}\right)(1-m)}, z^{*}=\frac{\left(\omega_{2}+(1-m) x^{*}\right)\left(1-x^{*}\right)}{c_{2}(1-m)}$,

$$
\bar{x}=\frac{d_{1} \varepsilon_{1}}{\left(e_{1} c_{1}-d_{1}\right)(1-m)}, \bar{y}=\frac{(1-x)\left(c_{1}+(1-m) x_{2}\right)}{c_{1}(1-m)} .
$$

The equilibrium point $E_{2}\left(x^{*}, 0, z^{*}\right)$ exists when

$$
c_{2} e_{2}>d_{2} \text { and } m<1-\frac{d_{2} \alpha_{2}}{c_{2} \varepsilon_{2}-d_{2}}
$$

The equilibrium point $E_{3}(\bar{x}, \bar{y}, 0)$ exists when

$$
c_{1} e_{1}>d_{1} \text { and } m<1-\frac{d_{1} \varepsilon_{1}}{e_{1} e_{1}-d_{1}}
$$

3 . The co-existence equilibrium point $E_{4}(\tilde{x}, \tilde{y}, \tilde{z})$, where

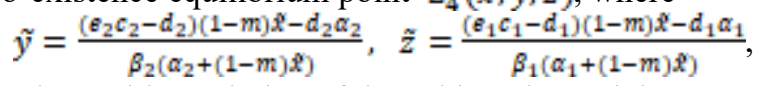

and $\tilde{x}$ is the positive solution of the cubic polynomial

with

$$
A_{3} \tilde{x}^{a}+A_{2} \tilde{x}^{2}+A_{1} \tilde{x}+A_{0}=0,
$$

$$
\begin{aligned}
& A_{2}=(1-m)^{2} \beta_{1} \beta_{2} A_{2}=(1-m)\left(\alpha_{1}+\alpha_{2}-(1-m)\right) \beta_{1} \beta_{2 x} \\
& A_{1}=\beta_{1}(1-m)\left(c_{1}\left(e_{2} c_{2}-d_{2}\right)(1-m)-\beta_{2}\left(\alpha_{1}+\alpha_{2}\right)\right)+\beta_{1} \beta_{2} \alpha_{1} \alpha_{2} \\
& +\beta_{2} c_{2}(1-m)^{2}\left(e_{1} c_{1}-d_{1}\right), \\
& A_{0}=(1-m)\left(c_{1} d_{2} \alpha_{2} \beta_{1}+c_{2} d_{1} \alpha_{1} \beta_{2}\right)+\alpha_{1} \alpha_{2} \beta_{1} \beta_{2} .
\end{aligned}
$$

Therefore, sufficient conditions for the existence of the positive equilibrium point in the interior of the first octant are easily obtained as follows:

$$
0<\tilde{x}<\min \left\{\frac{\alpha_{1} d_{1}}{\varepsilon_{1} \varepsilon_{1}-d_{1}}, \frac{\sigma_{2} d_{2}}{\varepsilon_{2} c_{2}-d_{2}}\right\}, m<1-\max \left\{1_{s} \frac{\beta_{2}}{c_{1}\left(\varepsilon_{2} c_{2}-d_{2}\right)}\right\}\left(\alpha_{1}+\alpha_{2}\right)
$$

\section{Local stability of the equilibrium points}

The local asymptotic stability of each equilibrium point is studied by computing the Jacobian matrix and checking the sign of the real part of the 
eigenvalues of the Jacobean matrix evaluated at each equilibrium point. For stability of the equilibrium points, the real parts of the eigenvalues of the Jacobian matrix must be negative.

The Jacobian matrix is

$$
l\left(E_{i}\right)=\left(\begin{array}{lll}
a_{11} & a_{12} & a_{12} \\
a_{21} & a_{22} & a_{23} \\
a_{21} & a_{22} & a_{22}
\end{array}\right),
$$

Where $a_{11}=1-2 x-\frac{c_{1} \omega_{1}(1-m) y}{\left(\alpha_{1}+(1-m) x\right)^{2}}-\frac{c_{2} \alpha_{2}(1-m) z}{\left(\alpha_{2}+(1-m) x\right)^{2^{x}}} \quad a_{12}=-\frac{c_{1}(1-m) x}{\alpha_{1}+(1-m) x^{x}}$ $a_{13}=-\frac{c_{2}(1-m) x}{\alpha_{2}+(1-m) x^{x}} \quad a_{21}=\frac{e_{1} c_{1} \alpha_{1}(1-m) y}{\left(\alpha_{1}+(1-m) x\right)^{2}}, \quad a_{22}=\frac{e_{1} c_{1}(1-m) x}{a_{1}+(1-m) x}-\beta_{1} z-d_{1}$, $a_{23}=-\beta_{1} y, a_{31}=\frac{e_{2} c_{2} a_{2}(1-m) z}{\left(a_{2}+(1-m) x\right)^{2}}, a_{32}=-\beta_{2 x}, a_{33}=\frac{\varepsilon_{2} c_{2}(1-m) x}{a_{2}+(1-m) x}-\beta_{2} y-d_{2}$.

One can see that the trivial equilibrium point $E_{0}(0,0,0)$ is always unstable.

Theorem 2: The axial equilibrium point $\mathrm{E}_{1}$ is stable provided

$$
m>1-\min \left\{\frac{d_{1} E_{1}}{\varepsilon_{1} c_{1}-d_{4}}, \frac{d_{2} \epsilon_{2}}{\varepsilon_{2} c_{2}-d_{2}}\right\} \text {. }
$$

Proof. The characteristic equation at $E_{1}(1,0,0)$ is

$(-1-\lambda)\left(\chi_{1}-\lambda\right)\left(\chi_{2}-\lambda\right)=0$, where

$\chi_{1}=\frac{e_{1} c_{1}(1-m)}{\alpha_{1}+(1-m)}-d_{1} \cdot \chi_{2}=\frac{e_{2} c_{2}(1-m)}{a_{2}+(1-m)}-d_{2}$.

Thus, all the eigenvalues are negative if $m>1-\min \left\{\frac{d_{1} a_{1}}{\varepsilon_{1} c_{1}-d_{1}}, \frac{d_{2} \alpha_{2}}{\varepsilon_{2} C_{2}-d_{2}}\right\}$.

Hence, the equilibrium point $E_{1}(1,0,0)$ is locally asymptotically stable.

Theorem 3: The axial equilibrium point $\mathrm{E}_{2}\left(\mathrm{x}^{*}, 0, \mathrm{z}^{*}\right)$ is stable provided $c_{1}>\frac{\left(c_{2} d_{1} a_{1}-a_{2} \beta_{1}\right)\left(c_{2} \varepsilon_{2}-d_{2}\right)+c_{2} d_{1} d_{2} \omega_{2}}{c_{2} d_{2} \varepsilon_{1} a_{2}} \times m>1-\frac{2 \varepsilon_{2} \varepsilon_{2} a_{2}}{\left(c_{2} \varepsilon_{2}-d_{2}\right)\left(c_{2} \varepsilon_{2}-d_{2}+1\right)}$.

Proof. The characteristic equation at $E_{2}$ is $\left(\Omega_{2}-\lambda\right)\left(\lambda^{2}+\Omega_{1} \lambda+\Omega_{0}\right)=0$, where

$$
\begin{gathered}
\Omega_{2}=-\frac{1}{c_{2} \varepsilon_{2}-d_{2}}\left(c_{1}-\chi_{1}\right)-\left(1-x^{*}\right)\left((1-m) x^{*}+\alpha_{1}\right)-\beta_{1}(1-m) x^{*}, \\
\Omega_{1}=\left(\frac{2}{(1-m) x^{*}+a_{2}}\right)\left(\left(c_{2} \varepsilon_{2}-d_{2}\right)\left(c_{2} e_{2}-d_{2}+1\right) \chi_{2}+d_{2} a_{2}\right), \\
\Omega_{0}=\frac{(1-m)\left(c_{2} \varepsilon_{2}-d_{2}\right)\left(1-x^{*}\right) x^{*}}{(1-m) x^{*}+c_{2}}, \\
\chi_{1}=\frac{\left(c_{2} d_{1} a_{1}-a_{2} \beta_{1}\right)\left(c_{2} \varepsilon_{2}-d_{2}\right)+c_{2} d_{1} d_{2} a_{2}}{c_{2} d_{2} \varepsilon_{1} a_{2}}, \chi_{2}=m-\left(1-\frac{2 c_{2} \varepsilon_{2} a_{2}}{\left(c_{2} \varepsilon_{2}-d_{2}\right)\left(c_{2} \varepsilon_{2}-d_{2}+1\right)}\right) .
\end{gathered}
$$

The eigenvalues are negative if and only if $\Omega_{2}<0, \Omega_{1}>0$ and $\Omega_{0}>0$. Since $\Omega_{0}>0$, one can easily see that $\Omega_{2}<0$ and $\Omega_{1}>0$ if condition (10) 
holds. Thus, $E_{2}\left(x^{*}, 0, z^{*}\right)$ is locally asymptotically stable.

Theorem 4: The axial equilibrium point $E_{3}(\bar{x}, \overline{\mathbf{y}}, 0)$ is locally asymptotically stable provided

$$
c_{2}>\frac{a_{2}\left(c_{1} d_{2}-\beta_{2}\right)\left(c_{1} e_{1}-d_{1}\right)+c_{1} d_{1} d_{2} e_{1}}{c_{1} d_{1} e_{2} \varepsilon_{1}}, m>1-\frac{2 e_{1} e_{1} \varepsilon_{1}}{\left(c_{1} e_{1}-d_{1}\right)\left(c_{1} e_{1}-d_{1}+1\right)} .
$$

Proof. The characteristic equation at $E_{0}$ is $\left(\gamma_{2}-\lambda\right)\left(\lambda^{2}+\gamma_{1} \lambda+\gamma_{0}\right)=0$, where $\gamma_{2}=-\frac{1}{c_{1} \varepsilon_{1}-d_{1}}\left(c_{2}-V_{1}\right)-(1-\bar{x})\left((1-m) \bar{x}+\alpha_{2}\right)-\beta_{2}(1-m) \bar{x}$, $\gamma_{1}=\left(\frac{2}{(1-m) x+a_{1}}\right)\left(\left(c_{1} e_{1}-d_{1}\right)\left(c_{1} e_{1}-d_{1}+1\right) V_{2}+d_{1} \alpha_{1}\right)$, $\gamma_{0}=\frac{(1-m)\left(c_{1} e_{1}-d_{1}\right)(1-x) x}{(1-m) x+a_{1}}$,

$V_{1}=\frac{a_{2}\left(c_{1} d_{2}-\beta_{2}\right)\left(c_{1} e_{1}-d_{1}\right)+c_{1} d_{1} d_{2} \varepsilon_{1}}{c_{1} d_{1} \varepsilon_{2} \varepsilon_{1}}, V_{2}=m-\left(1-\frac{2 c_{1} \varepsilon_{1} \varepsilon_{1}}{\left(c_{1} \varepsilon_{1}-d_{1}\right)\left(c_{1} \varepsilon_{1}-d_{1}+1\right)}\right)$.

The eigenvalues are negative if and only if $\gamma_{2}<0, \gamma_{1}>0$ and $\gamma_{0}>0$.

Since $\gamma_{0}>0$, one can easily see that $\gamma_{2}<0$ and $\gamma_{1}>0$ if condition (11) holds. Thus, $E_{a}(\bar{x}, \bar{y}, 0)$ is locally asymptotically stable.

Theorem 5: Suppose that the positive equilibrium point $\mathrm{E}_{4}(\tilde{\mathbf{x}}, \tilde{\mathbf{y}}, \mathbf{z})$ exists in the interior of the positive octant. Then $\mathrm{E}_{4}(\tilde{\mathrm{x}}, \tilde{\mathrm{y}}, \tilde{z})$ is not stable.

Proof. The entries of the Jacobian matrix evaluated at the positive equilibrium point $E_{4}$ are

$$
\begin{aligned}
& a_{11}=\left(-1+\frac{c_{1}(1-m)^{2} \tilde{y}}{\left(\alpha_{1}+(1-m) \tilde{x}\right)^{2}}+\frac{c_{2}(1-m)^{2} \tilde{z}}{\left(\alpha_{2}+(1-m) \tilde{x}\right)^{2}}\right) \tilde{x}_{v} \quad a_{22}=0, a_{a 3}=0, \\
& a_{12}=-\frac{c_{1}(1-m) \dot{s}}{a_{1}+(1-m) g}<0, a_{13}=-\frac{c_{2}(1-m) g}{a_{2}+(1-m) g}<0, a_{21}=\frac{e_{1} c_{1} a_{1}(1-m) g}{\left(a_{1}+(1-m) g\right)^{2}}>0 \text {, } \\
& a_{2 \mathrm{a}}=-\beta_{1} \tilde{y}<0, a_{31}=\frac{e_{2} c_{2} \alpha_{2}(1-m) \tilde{z}}{\left(\alpha_{2}+(1-m) \tilde{x}\right)^{2}}>0, a_{32}=-\beta_{2} \tilde{z}<0 .
\end{aligned}
$$

The characteristics equation of the Jacobian matrix around $E_{4}$ is:

$$
\lambda^{3}+A \lambda^{2}+B \lambda+C=0,
$$

Where $A=-a_{11}, B=-a_{23} a_{32}-a_{12} a_{21}-a_{13} a_{31}$

$C=a_{11} a_{23} a_{32}-a_{21} a_{32} a_{13}-a_{12} a_{23} a_{31}$. According to Routh-Hurwitz criteria, $E_{4}\left(x^{*}, y^{*}{ }^{*} z^{*}\right)$ is local asymptotic stability if and only if $A_{x} C$ and $A B-C$ are all positive.

Let $a_{11}>0$, then it can be seen that $A<0$. If $a_{11}<0$, then from the signs of Jacobean matrix elements $a_{i j},\left(i_{s} j=1,2,3\right)$ one can conclude that $C<0$. Therefore, the Routh-Hurwitz criteria is not satisfied and the positive equilibrium point $E_{4}$ is not stable. 


\section{Numerical simulation}

The dynamical behavior of the system (3) about the equilibrium points has been seen in the previous sections and obtained the analytical results. Now in this section, we have performed some numerical simulations by using MatLab to observe and describe the effect of prey refuge on the dynamics of the system (3) with a set of reasonable parameter values given in table (1). The effect of prey refuge on the dynamics of the system (3) is numerically investigated by keeping all parameters in table (1) constant except the prey refuge $m$.

Table 1. Parameter values of the system (3).

\begin{tabular}{lcc}
\hline Parameter & Value & Source \\
\hline$c_{1}$ & 0.82 & (Edwin, 2010) \\
$c_{2}$ & 0.86 & (Edwin, 2010) \\
$e_{1}$ & 0.085 & Estimated \\
$e_{2}$ & 0.086 & Estimated \\
$\alpha_{1}$ & 0.46 & Estimated \\
$\alpha_{2}$ & 0.48 & Estimated \\
$\beta_{1}$ & 0.01 & Edwin, 2010) \\
$\beta_{2}$ & 0.01 & Edwin, 2010) \\
$d_{1}$ & 0.02 & Estimated \\
$d_{2}$ & 0.02 & Estimated \\
$m$ & 0.2 & Estimated \\
\hline
\end{tabular}

\section{The effect of prey refuge $m$ to the equilibrium point $E_{2}\left(x^{*}, 0, z^{*}\right)$}

The equilibrium point $E_{2}\left(x^{*}, 0, z^{*}\right)$ exists for $m<0.82209$ and the rest of the parametric values are given in Table 1. The system (3) is locally asymptotically stable around the equilibrium point $E_{2}\left(x^{*}, 0, z^{*}\right)$ for $0.164181<m<0.82209$ and unstable otherwise. The existence condition (5) and the stability condition (10), (C.F. Theorem 3), are satisfied. The parametric plot of the solution of the system (3) in Figure 1 (top) shows a limit cycle about the equilibrium point $E_{2}(0.19337996,0,0.67073185)$ with parameter values as in Table 1 except $m=0.08$ in the $x-z$ plane. The time series solution of the system (3) in Figure 1 (bottom) shows that the system oscillates around the equilibrium point $E_{2}$ in the given time interval with parameter value as in Table 1. Thus, for the given set of parametric values as in Table 1 except for $m=0.08$, the equilibrium point $E_{2}$ is unstable. 
Figure 2 (top and bottom) represent the phase portrait and time series solution of the system (3) at the equilibrium point $E_{2}(0.22598870,0,0.74340068)$ for the set of parametric values as in Table 1, respectively. From Figure 2 (top and bottom), we can observe that the equilibrium point $E_{2}$ is locally asymptotically stable for the parametric values as in Table 1 .
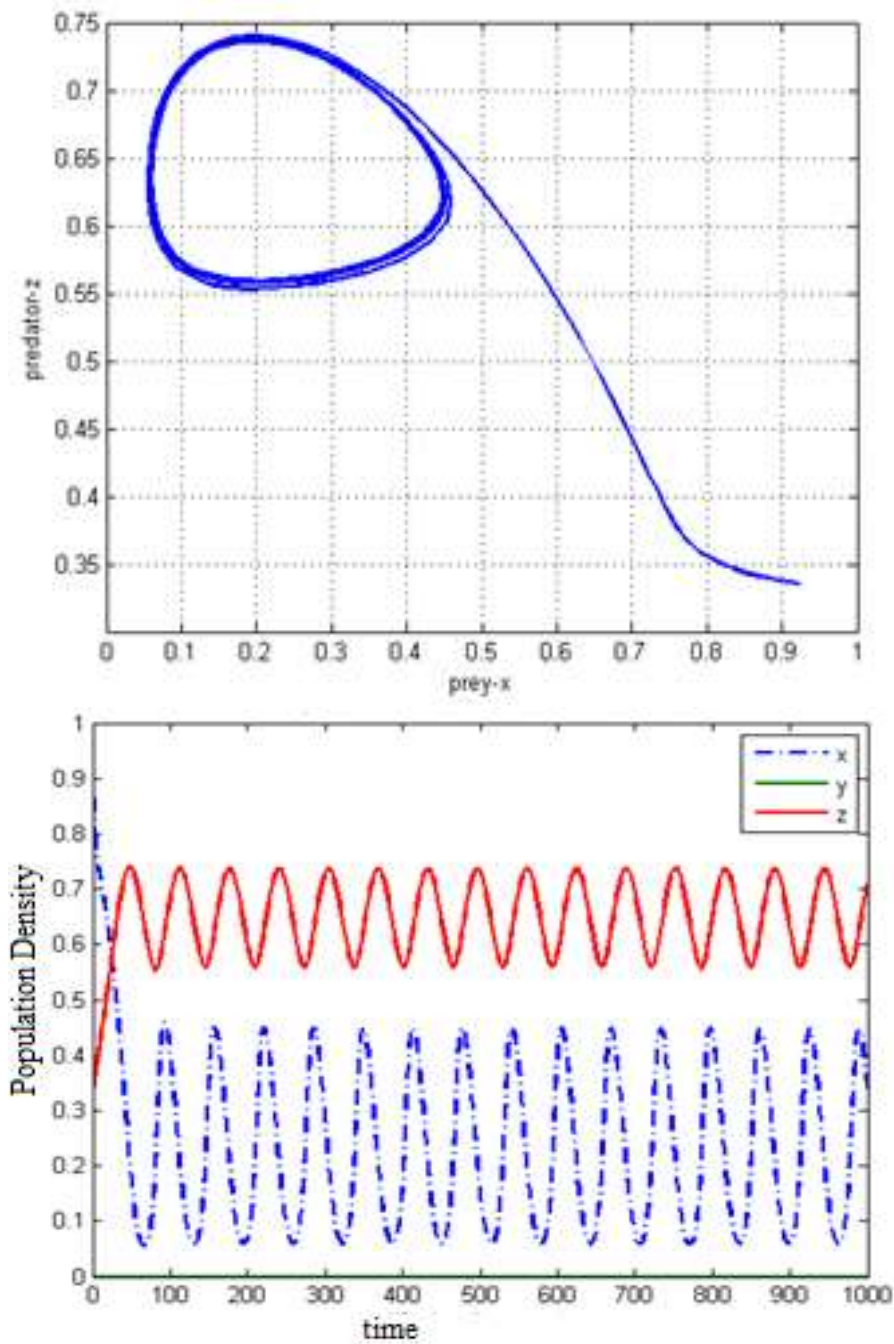

Figure 1. The parametric plot (top) and time series solution (bottom) of the system (3) about the equilibrium point $\mathrm{E}_{2}$ with parametric values as in Table 1 except $(\mathrm{m}=0.08)$. 

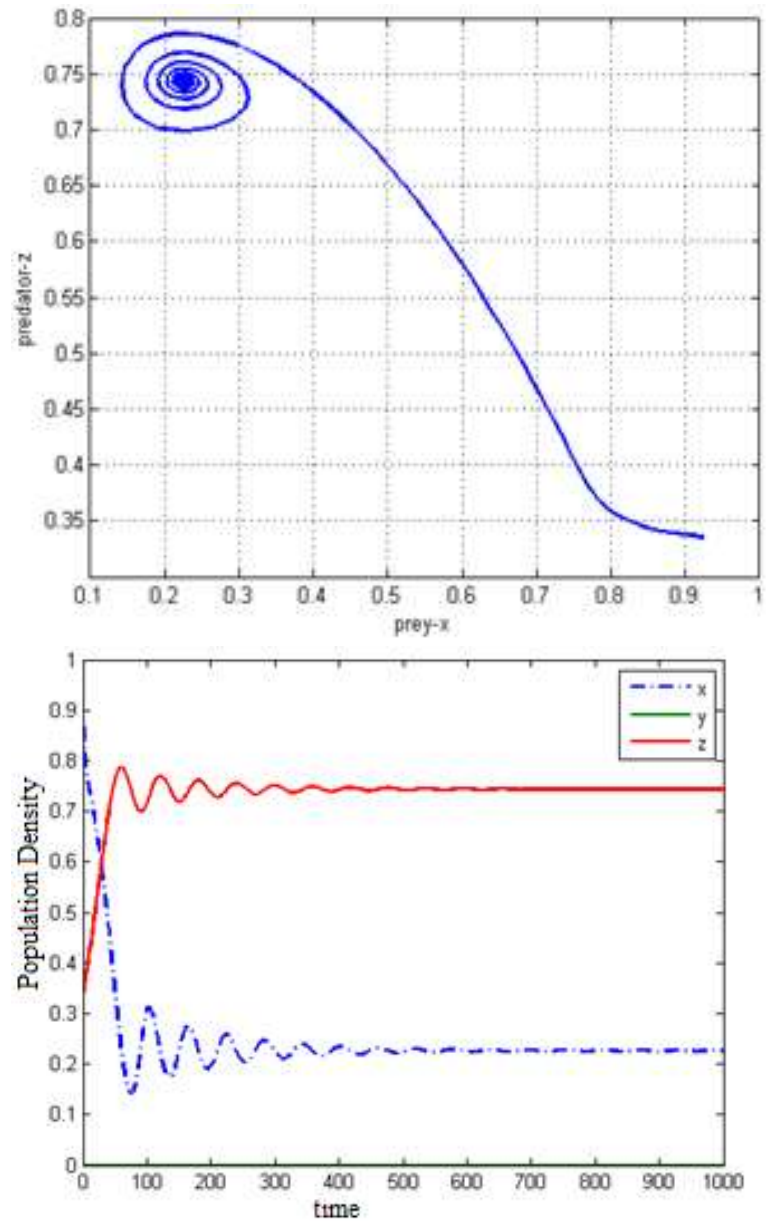

Figure 2. (Top) The phase portrait and (Bottom) time series of the system (3) around the equilibrium point $\mathrm{E}_{2}$ with parametric values as in table 1.

\section{The effect of prey refuge $m$ to the equilibrium point $E_{3}\left(\bar{x}_{*} \bar{y}_{v}, 0\right)$}

The equilibrium point $E_{3}\left(\bar{x}_{0}, \bar{y}, 0\right)$ exists for $m<0.814889$ and the rest of the parametric values are given as in Table 1. The system (3) is locally asymptotically stable around the equilibrium point $E_{a}\left(\bar{x}_{0}, \bar{y}, 0\right)$ for $0.169779<m<0.814889$ and unstable otherwise. The existence condition (6) and the stability condition (11), (C.F. Theorem 4), are satisfied. 
The phase portrait in Figure 3 (top) shows the limit cycle around the equilibrium point $E_{a}(0.21777725,0.72398886,0)$ with the parametric value in Table 1 except $m=0.15$. The time series solution in Figure 3 (bottom) shows an oscillation with high amplitude around the equilibrium point $E_{3}$ with the parametric value in Table 1 except $m=0.15$. Figure 3 (top and bottom) both signify that $E_{3}$ is unstable equilibrium point and the behavior of the system (3) is oscillatory.
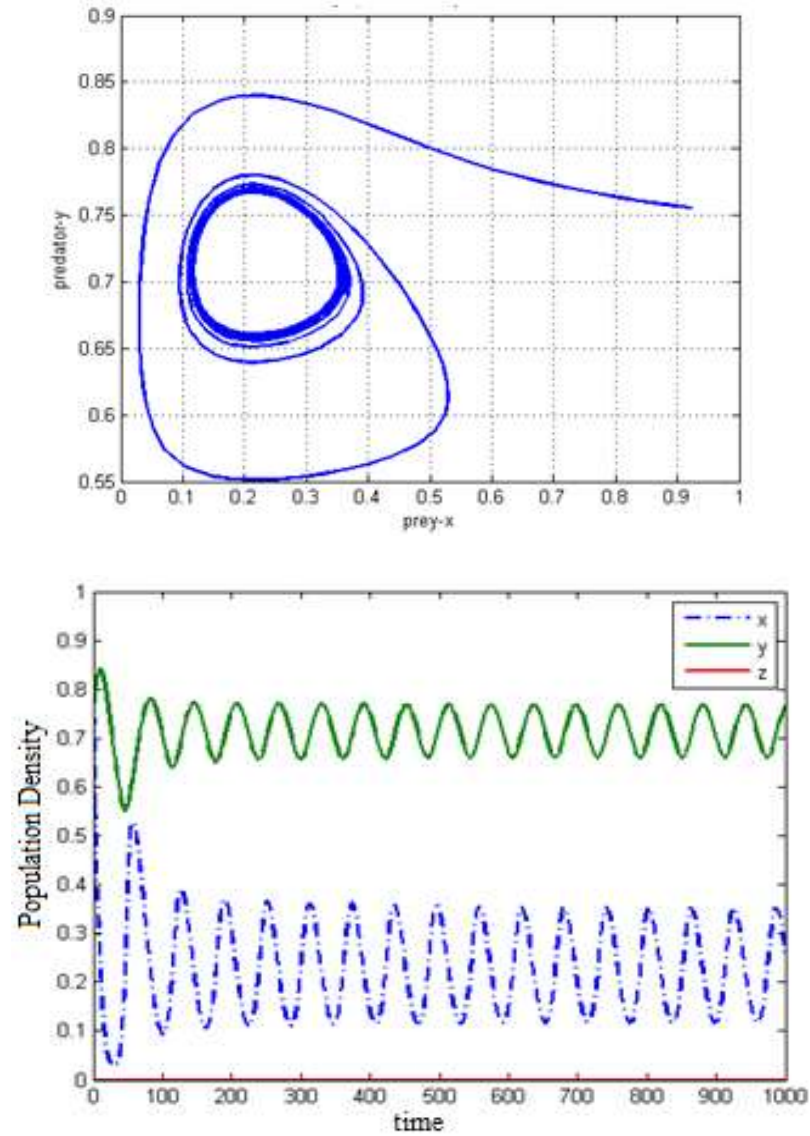

Figure 3. The phase portrait (top) and time series solution (bottom) of the system (3) around the equilibrium point $\mathrm{E}_{3}$ with parametric values as in Table 1 except $(\mathrm{m}=0.15)$.

The phase portrait in Figure 4 (top) and the time series solution (bottom) show that the equilibrium point $E_{3}(0.23138833,0.75585303,0)$ is locally 
asymptotically stable for the parameter values in Table 1 . Thus, in the absence of the predator-z the system (3) converges to the equilibrium point $E_{a}(0.23138833,0.75585303,0)$ for the parametric value as in Table 1.
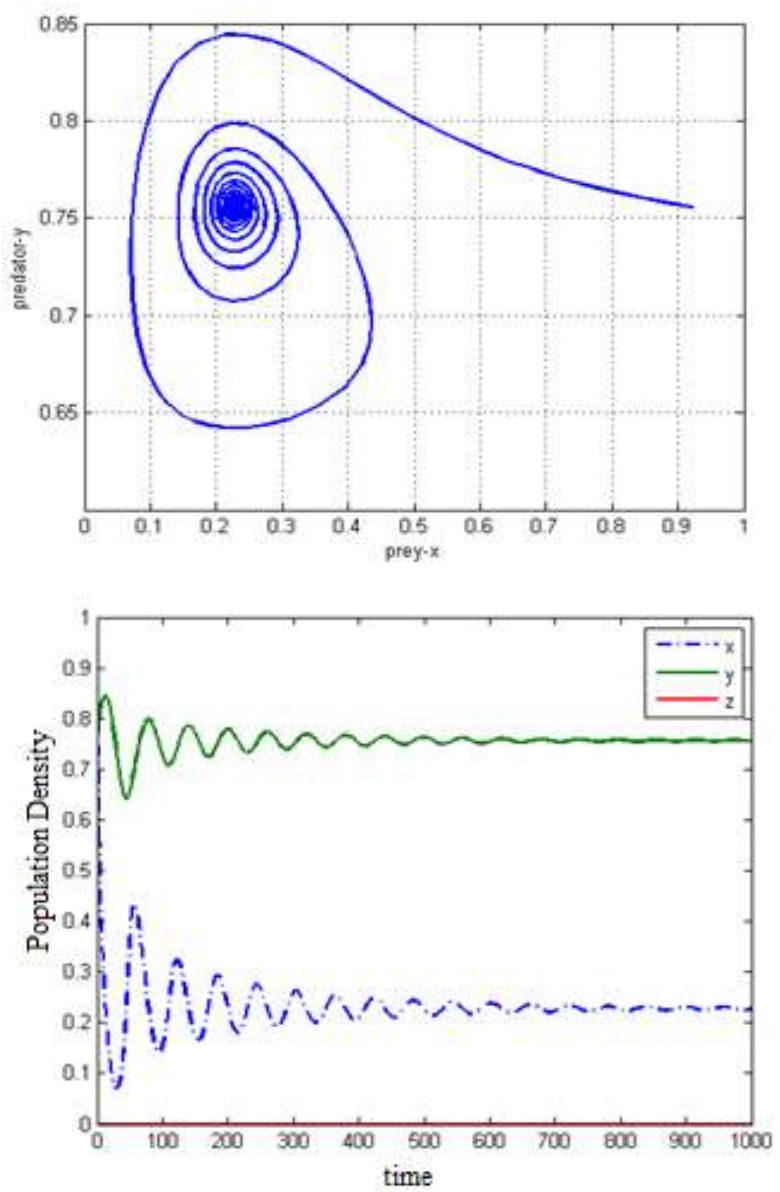

Figure 4. The phase portrait (top) and time series solution (bottom) of the system (3) around the equilibrium point $\mathrm{E}_{\mathfrak{g}}$ with parametric values as in Table 1 . 
The effect of prey refuge to the co-existence equilibrium point $E_{4}\left(\widetilde{x_{s}}, \widetilde{y}, \tilde{z}\right)$

The positive equilibrium point $E_{4}$ exists for $m<0.8122699$ and the rest of the parametric values are given as in Table 1. The time series solution in Figure 5 shows that for the given set of parametric values as in Table 1 except $(m=0.07)$, the prey and the predators oscillate around the positive equilibrium point $E_{4}(0.24389215,0.37338956,0.30181707)$. It is evident to see that all the three species persist for $m<0.164181$.

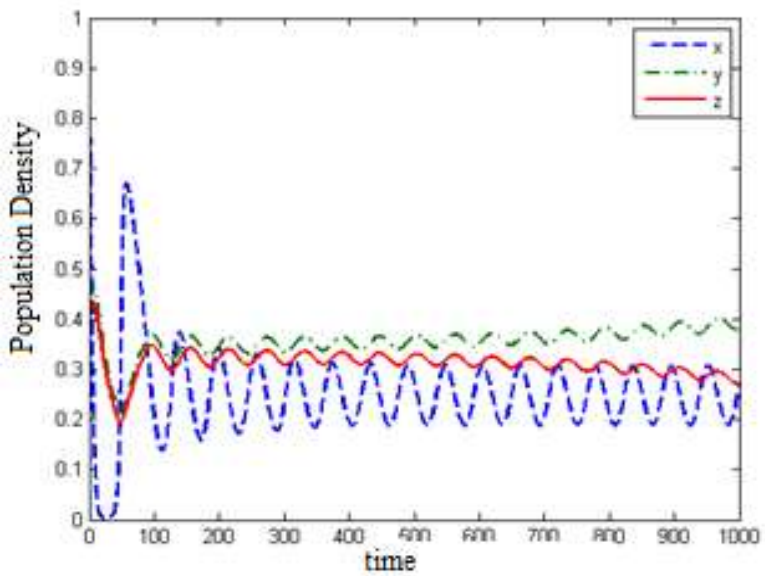

Figure 5. The time series solution of the system (3) around the positive equilibrium point $E_{4}$ with parametric values as in table 1 except $(m=0.07)$.

\section{CONCLUSION}

In this paper, a mathematical model is proposed and analyzed to study the effect of prey refuge on the dynamics of a three species food web system consisting of two predators competing for a single prey. Both predators show a Holling type II functional response to the prey. All the biologically feasible equilibrium points were calculated. The boundedness and positivity of the solutions of the system (3) are obtained. Conditions for the local stability of each equilibrium points, except the positive equilibrium point, are given with the help of the Routh-Hurwiz criteria.

Numerical simulations are performed to support and verify our analytical findings. In achieving this, we have concentrated on the equilibrium points, 
namely $E_{2}, E_{3}$ and $\mathrm{E}_{4}$. These observations depend on the parameter values in Table 1 except the prey refuge constant $m$, where $m$ is taken as a control parameter. The refuge constant is found to play a crucial role in stabilizing the dynamics of the system (3). Huang et al. (2006) stated that the non-zero equilibrium point could change from unstable state surrounded by a stable limit cycle to globally asymptotically stable state as increasing the prey in the refuge. Our numerical simulation shows also that an increase in prey refuge will lead to an extinction of one of the predators (see Figures 1 to 4 ).

From the numerical simulations we found that the system (3) is not persistent for $0.164181<m<0.8122699$. This is due to the fact that the system (3) has a stable boundary equilibrium points, $E_{2}$ and $E 3$, under this range. The principle of competitive exclusion holds in such cases. However, it is observed, c.f. Figure 5, that the system (3) exhibits persistence for $m<0.164181$, as the system (3) shows oscillatory behavior around the boundary equilibrium points $E_{2}$ and $E 3$. Therefore, one of the predators manages to survive at a very low level leading to persistence. Generally, as we have seen from the numerical simulation, as the number of preys in the refuge increases, the dynamics of a three species system changes the stability from limit cycle to a stable equilibrium point.

\section{ACKNOWLEDGMENTS}

The authors would like to thank the anonymous reviewers of this paper and the Editor-in-Chief for their supportive comments.

\section{CONFLICT OF INTEREST}

The authors declare that there is no conflict of interest in the publication of this paper.

\section{REFERENCES}

Alebraheem, J and Abu-Hasan, Y. (2012). Persistence of predators in a two predators-one prey model with non-periodic solution. Applied Mathematical Sciences 6(19):943-956.

Berryman, A.A. (1992). The origins and evolution of predator-prey theory. Ecology 73(5): 1530-1535.

Collings, J.B. (1995). Bifurcation and stability analysis of a temperature-dependent mite predator-prey interaction model incorporating a prey refuge. Bulletin of 
Mathematical Biology 57(1): 63-76.

Dubey, B and Upadhyay, R.K. (2004). Persistence and extinction of one-prey and two-predators system. Nonlinear Analysis: Modelling and Control 9(4): 307-329.

Edwin, A. (2010). Modeling and analysis of a two prey-one predator system with harvesting. Holling Type II and Ratio-dependent Responses, Master of Science in Mathematics of Makerere University, Uganda.

Freedman, H.I and Waltman, P. (1984). Persistence in models of three interacting predator-prey populations. Mathematical Biosciences 68(2): 213-231.

Gakkhar, S., Singh, B and Naji, R.K. (2007). Dynamical behavior of two predators competing over a single prey. Biosystems 90(3): 808-817.

González-Olivares, E and Ramos-Jiliberto, R. (2003). Dynamic consequences of prey refuges in a simple model system: more prey, fewer predators and enhanced stability. Ecological Modelling 166(1-2): 135-146.

Hassell, M.P. (2020). The dynamics of arthropod predator-prey systems (MPB-13), Volume 13. Princeton University Press.

Hsu, S.B. (1982). On a resource based ecological competition model with interference. Journal of Mathematical Biology 12(1): 45-52.

Hsu, S.B. Ruan, S and Yang, T.H. (2015). Analysis of three species Lotka-Volterra food web models with omnivory. Journal of Mathematical Analysis and Applications 426(2): 659-687.

Huang, Y., Chen, F and Zhong, L. (2006). Stability analysis of a prey-predator model with Holling type III response function incorporating a prey refuge. Applied Mathematics and Computation 182(1): 672-683.

Huo, H.F., Ma, Z.P and Liu, C.Y. (2009). Persistence and stability for a generalized Leslie-Gower model with stage structure and dispersal. In Abstract and Applied Analysis 2009. Hindawi.

Kar, T.K. (2005). Stability analysis of a prey-predator model incorporating a prey refuge. Communications in Nonlinear Science and Numerical Simulation 10(6): 681-691.

Ko, W and Ryu, K. (2006). Qualitative analysis of a predator-prey model with Holling type II functional response incorporating a prey refuge. Journal of Differential Equations 231(2): 534-550.

Krivan, V. (1998). Effects of optimal antipredator behavior of prey on predator-prey dynamics: the role of refuges. Theoretical Population Biology 53(2): 131-142.

Kuang, Y and Beretta, E. (1998). Global qualitative analysis of a ratio-dependent predator-prey system. Journal of Mathematical Biology 36(4): 389-406.

Li, H., Jiang, Y., Zhang, L and Teng, Z. (2014). Global stability for a three-species food chain model in a patchy environment. Journal of Applied Mathematics, 2014.

Lv, S and Zhao, M. (2008). The dynamic complexity of a three species food chain model. Chaos, Solitons and Fractals 37(5): 1469-1480.

McNair, J.N. (1986). The effects of refuges on predator-prey interactions: a reconsideration. Theoretical Population Biology 29(1): 38-63.

Mishra, P and Raw, S.N. (2019). Dynamical complexities in a predator-prey system involving teams of two prey and one predator. Journal of Applied Mathematics and Computing 61(1): 1-24.

Naji, R.K and Balasim, A.T. (2007). Dynamical behavior of a three species food chain 
model with Beddington-DeAngelis functional response. Chaos, Solitons and Fractals 32(5): 1853-1866.

Panday, P., Pal, N., Samanta, S and Chattopadhyay, J. (2018). Stability and bifurcation analysis of a three-species food chain model with fear. International Journal of Bifurcation and Chaos 28(01):1850009.

Reddy, K.S and Ramacharyulu, N.C.P. (2011). A three species ecosystem comprising of two predators competing for a prey. Advances in Applied Science Research 2(3): 208-218.

Sih, A. (1987). Prey refuges and predator-prey stability. Theoretical Population Biology 31(1): 1-12.

Smith, J.M. (1978). Models in Ecology excerpts. Cambridge University Press, London, first edition.

Srinivasu, P.D.N and Gayatri, I.L. (2005). Influence of prey reserve capacity on predator-prey dynamics. Ecological Modelling 181(2-3): 191-202.

Upadhyay, R.K and Naji, R.K. (2009). Dynamics of a three species food chain model with Crowley-Martin type functional response.Chaos, Solitons and Fractals 42(3): 1337-1346.

$\mathrm{Yu}, \mathrm{H}$ and Zhao, M. (2009). Dynamic behavior of a three-species ecological system with the Beddington-DeAngelis functional response. In 2009 International Workshop on Chaos-Fractals Theories and Applications: 346-350. IEEE. 